\title{
Más allá del negacionismo del cambio climático. Retos conceptuales al comunicar la obstrucción de la acción climática
}

\author{
Beyond climate change denialism. Conceptual challenges in \\ communicating climate action obstruction
}

\author{
Dra. Núria Almiron \\ Universitat Pompeu Fabra | Roc Boronat, 138, 08018 Barcelona | España | \\ https://orcid.org/0000-0002-2482-0666 | nuria.almiron@upf.edu
}

\author{
Jose A. Moreno \\ Universitat Pompeu Fabra | Roc Boronat, 138, 08018 Barcelona | España | \\ https://orcid.org/0000-0003-3781-5644 | joseantonio.moreno@upf.edu
}

\section{Resumen}

El cambio climático ha sido objeto de estudio frecuente desde distintos ámbitos de las ciencias sociales en las últimas décadas, también desde la comunicación. Gracias a ello, se ha generado una cada vez más abundante evidencia de la realidad compleja que se esconde tras la inacción política al respecto. Sin embargo, a la hora de comunicar esta realidad, los medios de comunicación, y una parte de la academia, han tendido a simplificar esta complejidad poniendo el foco en el contramovimiento climático y el negacionismo literal del fenómeno. Este contramovimiento ha sido abundantemente estudiado en los Estados Unidos, lo que ha puesto en evidencia la existencia de un negacionismo del cambio climático antropogénico muy influyente en ese país. Pero la investigación académica ha hecho también evidente que la inacción política al respecto del clima no puede explicarse solo con el concepto de negacionismo; ni en los Estados Unidos, ni mucho menos en Europa. En este artículo problematizamos el uso del concepto negacionismo en su uso indiscriminado actual y sugerimos incorporar un marco conceptual y de análisis más sofisticado, que aporte más matices y se alinee

\section{Abstract}

Climate change has been the subject of much research in various fields of the social sciences in recent decades, including that of communication. As a result, much evidence has been accumulated on the complex reality behind political inaction in this regard. However, when it comes to communicating this reality, the media, and part of academia, have tended to simplify this complexity by focusing on the climate countermovement and literal denialism of the phenomenon. This countermovement has been extensively studied in the United States, revealing the existence of a highly influential anthropogenic climate change denialism in that country. However, academic research has also shown that political inaction on the climate cannot be explained by denialism alone; not in the United States, and much less in Europe. In this article, we question the current indiscriminate use of the concept of denialism and suggest the incorporation of a more sophisticated conceptual and analytical framework that provides more nuance and aligns with the evidence emerging from academic research. It is a matter not only of critically communicating the reality of political inaction with regard to the climate, but also of 
con las evidencias de la investigación académica. Se trata no solo de comunicar de forma crítica la realidad de la inacción política con respecto al clima sino de hacerlo también identificando todo el espectro real de responsabilidades, que no se reducen a negar o no negar el cambio climático.

Palabras clave: cambio climático, Keywords: climate change, denialism, inaction, negacionismo, inacción, obstruccionismo, contrarianismo.

\section{INTRODUCCIÓN}

El término negacionismo, un concepto con una enorme carga ideológica, se ha incorporado al vocabulario habitual de los medios de comunicación, las redes sociales y el debate público. La crisis del COVID-19 ha contribuido a ello, pero el término se ha venido aplicando a otras cuestiones de forma frecuente desde hace tiempo, entre ellas el cambio climático. Cuando en 2017 iniciamos el proyecto de investigación THINKClima ${ }^{1}$, lo titulamos "Cambio climático, negacionismo y comunicación de influencia. Discurso y estrategias de los think tanks en Europa". Tras el bagaje de cinco años de trabajo, nuestra visión del negacionismo se ha transformado: nuestros resultados indican que generalizar la oposición a la lucha climática utilizando el término negacionismo es un error que trivializa un asunto muy delicado, el propio negacionismo, y conduce a engaño sobre el reparto real de responsabilidades en la crisis climática.

En este artículo defendemos que es necesario evitar un uso indiscriminado del término negacionismo al comunicar sobre la crisis climática. Basamos nuestra argumentación en que esta denominación impide identificar correctamente las causas de la inacción frente a la crisis climática debido a su carácter reduccionista. De hecho, esta etiqueta puede resultar en sí misma una ocultación de los problemas que explican la inacción climática. El campo académico que estudia la inacción política con respecto al calentamiento global ha estado evolucionando hacia otras denominaciones que describen mejor la realidad. Por este motivo, la investigación académica ha abandonado progresivamente el término negacionismo como categoría marco de la oposición a la lucha climática, para incorporar otras visiones, más precisas, que recogen las particularidades de las trabas interpuestas a la lucha climática. Estas nuevas denominaciones implican nuevos marcos conceptuales que incluyen, por ejemplo, el retardismo (delay), el contrarianismo (contrarianism) o el obstruccionismo (obstructionism).

En este artículo problematizamos el uso del concepto negacionismo, en su uso indiscriminado actual, y sugerimos incorporar un marco conceptual y de análisis más sofisticado, que aporte más matices y se alinee con las evidencias de la investigación académica. Se trata no solo de comunicar de forma crítica la realidad de la inacción política con respecto al medioambiente y el calentamiento global sino de hacerlo también identificando todo el espectro real de responsabilidades, que no se reducen a negar o no negar el cambio climático.

\footnotetext{
${ }^{1}$ Proyecto perteneciente al Programa Estatal de Investigación, Desarrollo e Innovación Orientada a los Retos de la Sociedad (CSO2016-78421-R). Más información en www.upf.edu/web/thinkclima
} 
Reconocemos la dificultad de nuestra propuesta. El término negacionismo es sencillo y atractivo; esto es, permite titulares y enfoques llamativos y tranquilizadores (la culpa recae en los negacionistas). Sin embargo, la comunicación de las crisis no debe ser solo crítica sino también honesta, evitando reduccionismos (por ejemplo, que polaricen u oculten a parte de los responsables) e interpretaciones interesadas (que por ejemplo achaquen toda la responsabilidad a un grupo concreto de actores, como en este caso a la derecha conservadora).

\section{MARCOS CONCEPTUALES MÁS ALLÁ DEL NEGACIONISMO}

El término negacionismo se ha utilizado comunicativamente como concepto paraguas para referirse a multitud de posiciones distintas no alineadas con la lucha contra el cambio climático. Sin embargo, no todos los no alineados con la lucha contra el cambio climático responden efectivamente a la definición de negacionistas, un concepto con un bagaje ideológico considerable, como decíamos. Esto es así porque el origen del término está, como no escapa a nadie, históricamente asociado a la negación del Holocausto judío durante la Alemania nazi. Por este motivo, muchas definiciones institucionales del término lo vinculan al revisionismo histórico y al derecho internacional. De hecho, en el Diccionario Panhispánico de Dudas puede leerse como definición de negacionismo: "Delito de odio que comete quien niegue públicamente un delito de genocidio, de lesa humanidad o contra las personas y bienes protegidos en caso de conflicto armado". El término merece pues una aplicación ajustada y ha sido por ello abiertamente problematizado por diversos autores antes de este artículo con respecto a su uso en el ámbito de la lucha climática (por ejemplo Jacques, 2012; O’Neill y Boykoff, 2010). Sin embargo, no ha sido hasta hoy analizado en profundidad como proponemos aquí.

El uso indiscriminado de negacionismo no es solo un problema de precisión históricosemántica. Su aplicación produce una división que, como veremos a continuación, es falaz, pues no existe algo así como dos bandos en la inacción climática (el negacionista y el no negacionista) sino un conglomerado de actores entre los que algunos niegan, bastantes más obstruyen y una enorme mayoría no colabora o boicotea la acción climática inconscientemente. En realidad, la etiqueta negacionismo acaba sirviendo para ocultar la responsabilidad que tienen los no negacionistas.

Pese a estos problemas, esta es la denominación que aparece con más frecuencia en medios de comunicación y en el debate público para hacer referencia a quienes se oponen a la acción climática, que son por lo general calificados de "negacionistas del cambio climático" (o incluso de "negacionistas climáticos", que supondría que niegan el clima, lo cual evidentemente no hace nadie). Con esta y otras aplicaciones que trivializan el concepto, vemos como negacionismo se utiliza como un contenedor que irreflexivamente se aplica a los disidentes en cualquier problema complejo. En el caso del cambio climático, en castellano se confunde además negacionismo (ideología, denialism) con negación (actitud, denial) - esta última un estado mental que no solo subyace en el negacionismo explícito, sino que también se encuentra entre los que no niegan el cambio climático literal pero sí sus implicaciones-. 
Sobre la negación como actitud han teorizado distintos investigadores. Entre ellos, de forma destacada, el sociólogo Stanley Cohen (2001) identificó tres estados de negación común entre las personas que experimentan eventos que causan sufrimiento: el literal, el interpretativo y el implicatorio. La clasificación de Cohen es de enorme utilidad para entender el fenómeno de la inacción climática. Aplicada a ella, la negación literal supone el rechazo a reconocer los hechos o la evidencia del calentamiento global. La negación interpretativa, en cambio, no niega los hechos que ocurren, pero les otorga un significado distinto a lo que para otras personas sería evidente - por ejemplo, no interpretar el calentamiento global actual como causado por el ser humano-. La negación implicatoria, por último, sí que asume la evidencia sobre el problema ante el que se encuentra, sin embargo, niega "las implicaciones psicológicas, morales o políticas" necesarias para reducir las emisiones de gases contaminantes (Cohen 2001, 8). Las dos primeras tipologías, implicación e interpretación, pueden llevar a construir la ideología del negacionismo del cambio climático, y han sido estudiadas ampliamente en los Estados Unidos con respecto a la oposición a la acción climática: hay personas y organizaciones que efectivamente niegan la evidencia del calentamiento global, y algunas más que niegan su carácter antropogénico, y que han incorporado este estado de negación a su ideario político. Sin embargo, estos dos estados de negación no implican siempre posicionamientos negacionistas: por ejemplo, se puede negar la severidad del problema, sin negar el problema en su conjunto, o se puede negar, como sucede a menudo, que podamos solventarlo. Etiquetar como negacionistas estas últimas dos posturas, por ejemplo, es maniqueo y produce confusión. Del mismo modo, tampoco implica negacionismo en la mayoría de casos el tercer estado de negación, la negación implicatoria, que de hecho es común entre las personas y organizaciones que reconocen y se preocupan por el cambio climático antropogénico - pero se niegan a aceptar el cambio de hábitos que ello supone-. Por ello, confundir la negación con el negacionismo es un reduccionismo que nos impide entender la complejidad real tras la inacción climática.

En concreto, sostenemos aquí que la utilización del término negacionismo para hacer referencia a aquellos actores que boicotean o que no secundan la acción climática no es adecuada como concepto paraguas por tres motivos: en primer lugar, porque la mayoría de los disidentes climáticos no niegan la ciencia climática, sino que se oponen a la adopción de políticas climáticas; en segundo lugar, porque genera una polarización que intoxica el debate público y no refleja realmente la composición del contramovimiento climático, y, finalmente, y vinculado a lo anterior, porque el término negacionismo invisibiliza a los que contribuyen a la inacción climática sin ser negacionistas $-y$ con ello invisibiliza a la base ideológica que los sustenta-.

\subsection{El obstruccionismo (negacionista o no) es la realidad dominante}

Tanto en los Estados Unidos como en Europa está claro que la tipología de los argumentos de las personas y grupos que disienten del activismo climático es mucho más amplia que lo que el término negacionista implica. Los negacionistas sin duda existen -aquellas personas que niegan la existencia o el consenso científico sobre el calentamiento global antropogénico-, pero no representan ni mucho menos todas las posturas en oposición. El análisis del ideario utilizado tanto en los Estados Unidos como en Europa muestra que los argumentos 
estrictamente negacionistas no son los que predominan en las narrativas contrarias a la acción climática (Almiron et al., 2020; Coan et al., 2021). Históricamente, desde que aparece la disidencia climática, los argumentos más utilizados son principalmente los relacionados con las soluciones políticas y el intento de desprestigio de los activistas climáticos.

En realidad, los datos históricos muestran que la enorme mayoría de los disidentes climáticos no niegan el calentamiento global, sino que esencialmente se oponen a las políticas que pretenden atajar el problema. Los motivos esgrimidos para esta oposición son sobre todo de ausencia de eficacia o incluso de prioridad - porque tenemos otros problemas más acuciantes o porque las políticas no pueden arreglar el problema, o incluso pueden empeorarlo, y además perjudican a la economía-. Obviamente, la causa subyacente a esta oposición es simplemente el intentar seguir con el business as usual, para que todo siga igual en aquellos negocios que implican más impacto medioambiental.

En general, lo que las investigaciones han desvelado es que hay algunos negacionistas de la crisis climática antropogénica, pero, sobre todo y eminentemente, hay obstruccionismo político. Por este motivo, los investigadores anglosajones han sido tan prolíficos en las etiquetas utilizadas, que han incluido hablar de negación que muchas veces se traduce como negacionismo-“maquinaria negacionista" (denial machine), el conjunto de actores que buscan poner en duda el consenso climático y atacan la ciencia climática (Dunlap y McCright, 2011)-, pero también, por ejemplo, de:

- escepticismo (skeptics), aquellos que dudan de la ciencia climática atribuyéndose un posicionamiento pseudocientífico (p.ej., Dunlap, 2013; Lahsen, 2013)

- contramovimiento (countermovement), término que define a aquellos actores que trabajan en armonía, pero sin necesaria coordinación para socavar la ciencia y las políticas climáticas (p.ej., Dunlap y McCright, 2015; McKie, 2017)

- contrarianismo (contrarians), utilizado para definir a aquellos que se oponen a la acción climática (p.ej., Boykoff, 2016; McCright, 2007)

- retardismo (delayers), cuyos discursos ponen en duda las medidas para frenar el cambio climático y promueven la inacción (Lamb et al., 2020), o, más recientemente

- obstruccionismo (obstructionism), término que describe a quienes de algún modo boicotean u obstruyen la acción política para reducir emisiones y contaminación en general (p.ej., CSSN, 2021; McKie, 2021).

Cada denominación refleja distintos matices, pero en nuestra opinión es la última, obstruccionismo, la que incorpora una posición mayoritariamente compartida por todos estos actores. Los obstruccionistas están unidos por el hecho de percibir como una amenaza cualquier alteración del statu quo que se oponga a sus intereses. Los vínculos con la derecha conservadora, el libertarismo y el neoliberalismo del contramovimiento climático en Estados Unidos y en Europa (Brulle, 2014; Jacques et al., 2008; Jylhä y Hellmer, 2020; McCright y Dunlap, 2010, 2011) lo señalan: no es el negacionismo lo que une a quienes se oponen a la acción climática, sino la defensa del capitalismo financiero, oligárquico y patriarcal. Es cierto que algunos son negacionistas, pero muchos otros son escépticos o simplemente van a la contra, mientras que prácticamente todos son defensores del statu quo económico y obstruyen las políticas climáticas. 
Así, el estudio académico de la disidencia en lo relativo al cambio climático ha dejado de estar centrado en el negacionismo para poner el foco en el obstruccionismo. El obstruccionismo es tanto una narrativa como una estrategia. En los dos casos el objetivo es el mismo: retrasar las políticas que pretenden reducir las emisiones de gases efecto invernadero o la contaminación en general por ser vistas como una amenaza a los negocios. La Climate Social Science Network define los esfuerzos obstruccionistas con estas tres acciones (CSSN, 2021):

1) Mantener o transformar la agenda pública para que se acepte la interpretación deseada como el sentido común en la cuestión, y garantizar que la opinión pública no apoya la acción climática.

2) Influir en la agenda mediática para promocionar perspectivas concretas que arrojen dudas sobre las acciones que pretenden abordar el cambio climático.

3) Influir en el proceso político para que se promuevan las políticas que no apoyan la acción climática.

Esta estrategia incluye argumentos retardistas como que no es posible mitigar el cambio climático y hemos de rendirnos; que las responsabilidades pertenecen a otros, que son quienes tienen que actuar primero; que no hace falta aplicar cambios profundos para combatir el cambio climático, o que los cambios y medidas necesarios tienen muchas desventajas (Lamb et al., 2020). Este tipo de argumentos, mucho más pragmáticos y menos hostiles a la evidencia científica, están más extendidos de lo que parece en el ámbito empresarial y económico, y ejercen gran influencia en el ámbito político -como muestra, basta ver cómo la retórica de la adaptación a los escenarios climáticos que se dan por inevitables ha sustituido ya a la lucha por la mitigación de las emisiones de efecto invernadero en escenarios como las cumbres climáticas organizadas por Naciones Unidas-.

En el ámbito empresarial, el obstruccionismo a la acción climática se lleva a cabo de forma mucho más contundente en la sombra, a través de corporaciones que han incorporado la retórica del activismo climático en su discurso público pero que en la práctica -en los corredores del poder - hacen lobby en contra de las políticas pro-ambientales. Esta dinámica lógicamente requiere de grandes dosis de relaciones públicas, para que la contradicción entre el discurso público y la acción en la sombra no se hagan evidentes. Es lo que se ha venido en llamar greenwashing - esto es, la utilización de estrategias de marketing verde para dar una imagen positiva y ambientalista que oculta una mala praxis con respecto a problemas medioambientales (de Freitas Netto et al., 2020)-. De entre los actores corporativos más obstruccionistas, que no negacionistas, destacan algunas industrias, principalmente de los sectores energético, del transporte y agrícola-alimentario, cuyos lobbies se han dedicado a retrasar tanto como han podido la acción climática.

Un caso ampliamente estudiado en los EE.UU. es el de la compañía Exxon, cuya estrategia de relaciones públicas ha estado destinada a difundir ideas negacionistas del cambio climático a través de think tanks y grupos de presión (Oreskes y Conway, 2018) y a través de campañas en medios de comunicación (Supran y Oreskes, 2017). De hecho, un análisis sobre la comunicación de esta compañía revela que su objetivo ha sido restar gravedad a la emergencia climática y culpar a los consumidores para así ocultar la responsabilidad que tiene la propia 
empresa (Supran y Oreskes, 2021). Dinámicas similares ocurren en contextos como el español, donde las empresas energéticas que se encuentran entre las que más gases de efecto invernadero emiten patrocinaron la COP25 Madrid-Chile y diversos contenidos en prensa para reverdecer su imagen durante la cumbre (Moreno y Ruiz-Alba, 2021).

Estas estrategias no se limitan al sector energético. Por ejemplo, para el caso del lobby de la carne en la Unión Europea es posible trazar una estrategia que replica las tácticas del obstruccionismo climático clásico: diseminar información que ponga en duda el consenso existente sobre el carácter contaminante de la producción de carne y convencer a los políticos que la tecnología (no un cambio de dieta) es la solución (Almiron, 2020). El lobby de la carne también se inserta en coaliciones discursivas contra la adopción de políticas climáticas (Almiron y Moreno, 2021), esto es, grupos que impulsan una cierta narrativa aunque no estén necesariamente coordinados, como ocurre con otros sectores en el seno del contramovimiento climático. En concreto, una de las estrategias con las que el lobby de la carne ha conseguido retrasar las decisiones políticas al respecto de las emisiones de la industria agrícola y alimentaria basada en animales ha sido reenfocando la discusión en aspectos que demoran indefinidamente las decisiones-por ejemplo, discutiendo y rediscutiendo cómo deben medirse las emisiones-. Con acciones de este tipo, este lobby, junto a los lobbies energético y del transporte y otros, han obstruido sistemáticamente la acción política climática, retrasando cualquier cambio relevante. Públicamente, todas estas industrias muestran preocupación y conciencia medioambiental (greenwashing), pero al mismo tiempo dedican ingentes cantidades de dinero y esfuerzos en hacer lobby para obstaculizar las políticas climáticas eficaces, que son vistas como una amenaza para sus negocios. Esta realidad, este obstruccionismo, no la influencia de los negacionistas que rechazan radicalmente el cambio climático antropogénico, es lo que ha impedido que en Europa se avance en materia de política medioambiental de forma eficaz, a pesar de ser una de las regiones del planeta donde sus habitantes están más concienciados en el tema según el Eurobarómetro (European Parliament, 2017).

\subsection{La polarización "negacionistas vs no negacionistas" es reduccionista (y engañosa)}

En los Estados Unidos el debate sobre el cambio climático ha estado tremendamente polarizado desde sus inicios (Rekker, 2021). Esta polarización estadounidense a menudo ha sido interpretada desde fuera con una mirada reduccionista, como si significara que la mitad de la población es negacionista mientras la otra mitad no lo es, y quedaría así exonerada de toda culpa en la inacción climática. Tal polarización no existe en Europa, donde la inmensa mayoría de la población se alinea con el consenso científico sobre el clima y donde, sin embargo, las políticas medioambientales necesarias para reducir emisiones contaminantes tampoco se implementan.

En este contexto, el uso del término negacionista no es simplemente una inexacta generalización aplicada al contramovimiento climático, sino también una estrategia retórica maniquea que abona esa falsa polarización del debate climático distinguiendo entre malos (negacionistas) y buenos (no negacionistas). Esto permite criminalizar a los primeros y exculpar a los segundos, cuando la realidad es que en el segundo grupo hay tantos o más obstruccionistas (aunque no sean negacionistas de las bases científicas del cambio climático). 
Los investigadores estadounidenses hace tiempo que se percataron de ello alertando que la disidencia a la lucha climática está formada por un grupo de actores mucho más amplio que la órbita de think tanks financiada por la industria petrolera (p.ej., CSSN, 2021; Dunlap y McCright, 2015). Además de estos, en Estados Unidos el contramovimiento incluye también a corporaciones y asociaciones comerciales, coaliciones y grupos de interés, agencias de relaciones públicas, grupos activistas de falsa bandera, filántropos y fundaciones conservadoras, algunos científicos, medios de comunicación, políticos conservadores y blogueros. En Europa, también hemos podido identificar que los think tanks obstruccionistas no están solos: están al menos conectados con el movimiento neoliberal (p.ej., Almiron et al., 2020), con el contramovimiento estadounidense (p.ej., Huth y Peters, 2020) y con campañas como la del Brexit (Farand et al., 2019), además de contar también con periodistas y blogueros afines. A este respecto, un estudio que realizamos sobre un centenar de los think tanks más importantes de Europa de todas las ideologías ha demostrado que estas organizaciones en Europa han invertido una atención mínima, anecdótica, con poquísimas excepciones, en la importante cuestión de la dieta basada en animales y su impacto en las emisiones de efecto invernadero (Almiron et al., 2021). Todo lo cual pone en evidencia que los que mantienen algún grado de retardismo o de no colaboracionismo son muchos más de lo que hemos venido en llamar negacionistas y asociamos con la derecha ideológica.

Otro aspecto sobre el que cabe reflexionar acerca del maniqueísmo que crea el uso del concepto negacionista es la heterogeneidad de los no negacionistas. Entre los no negacionistas no solo se encuentran organizaciones que no dudan de la existencia del cambio climático pero que por interés económico actúan para obstruir la acción climática. También se encuentran en este grupo organizaciones progresistas y la propia ciudadanía. Entre las primeras encontramos, por ejemplo, a las mismas organizaciones ecologistas, que en su lucha climática no han incorporado aspectos como la denuncia contra la dieta basada en animales hasta hace relativamente poco y no de forma unánime (Kramcksak Muñoz, 2021). En lo que respecta a la ciudadanía, si bien en Europa esta es mayoritariamente consciente del problema medioambiental, ello no significa que asuma y acepte los cambios de hábitos que se requieren. Entre los cambios de hábitos con mayor potencial de mitigación están el abandono del coche privado, el rechazo a los viajes en avión, el consumo de energía exclusivamente renovable en el hogar o la adopción de una dieta vegana (Ivanova et al., 2020). Negar el impacto de estas medidas por cuestiones culturales e ideológicas concuerda con la negación implicatoria tal como la describió Stanley Cohen (2013). En general, la ciudadanía europea es consciente del problema climático, no lo niega (European Parliament, 2017), pero sí niega las implicaciones de esos hechos, es decir, los cambios de conducta que han de ser tomados para ser consecuente con la situación de crisis climática. Esta negación busca sostener privilegios que la ciencia ha mostrado como medioambientalmente insostenibles pero que la ciudadanía justifica en base a argumentos comúnmente anclados en la tradición, la cultura o la ideología. En este sentido, la socióloga Kari Marie Norgaard (2011) achaca estas actitudes a la necesidad de mantener cierta "seguridad ontológica" (p. 26), esto es, continuidad con el estilo de vida y la certeza de un futuro estable. Así pues, paradójicamente, una gran parte de la ciudadanía se estaría refugiando también en una narrativa de obstruccionismo y retraso a la acción climática a pesar de su conciencia climática. 


\subsection{La crítica al negacionismo invisibiliza el statu quo antropocéntrico-patriarcal-industrial del obstruccionismo}

El concepto negacionismo usado como paraguas ideológico de la inacción climática no es solo una imprecisión reduccionista, es también una forma de invisibilizar el conjunto de ideas y valores antropocéntrico-patriarcales e industrialistas que subyacen en la inacción climática (tanto negacionista como no negacionista).

Hemos visto que en la obstrucción de la acción climática tienen un papel importante los intereses económicos de las industrias implicadas, así como las ideologías que defienden el libre mercado y posiciones materialistas. Esta dinámica está soportada por una cosmovisión antropocéntrica, industrial y patriarcal, un conjunto de creencias interconectadas que definen la cultura y forma de ver el mundo de la sociedad occidental.

Vivimos tiempos de incertidumbre marcados por la insostenibilidad, es decir, la forma en que funciona la sociedad ha de cambiar o colapsará (Garcés, 2017; Taibo, 2011). La noción antropocéntrica de progreso que guía a la humanidad ha estado tradicionalmente centrada en la acumulación de riqueza mediante la explotación de unos recursos naturales finitos y de los animales no humanos (Best, 2014; Hribal, 2014). Los procesos de acumulación que tienen lugar en el sistema económico se sirven del patriarcado para perpetuarse (Mies, 2018). Hay evidencias que vinculan esta noción de progreso con un cierto tipo de masculinidad denominada "industrial" (Hultman y Pulé, 2018), y que puede ser representativa del patriarcado occidental hegemónico. Esta masculinidad emana de las normas sociales relativas a lo que significa ser un hombre, y está profundamente ligada a los ideales de crecimiento industrial, las industrias extractivas y las prácticas dependientes de los combustibles fósiles (Hultman y Pulé, 2018). Diferentes investigaciones señalan que los hombres blancos conservadores occidentales, exponentes de la masculinidad "industrial", son los más propensos a oponerse a la acción climática o negar el cambio climático (Anshelm y Hultman 2014; McCright y Dunlap 2011; Krange et al. 2019). Además, la literatura ha demostrado que un pilar de esta masculinidad hegemónica es la aniquilación de otros animales (Adams, 2010). Diversos estudios han demostrado que los hombres son más propensos a justificar las dietas basadas en animales que las mujeres, debido a una identificación profunda entre la masculinidad y la carne (p.ej., Love y Sulikowski, 2018; Rothgerber, 2013). Incluso en el ámbito de la movilidad hay evidencias que vinculan esta masculinidad con la cultura del coche, siendo los automóviles objetos asociados al poder y la virilidad (Duerringer, 2015; Walker et al., 2000). Así, frenar el calentamiento global es controvertido ya que ataca pilares tan fundamentales para las masculinidades en crisis como lo son la carne, el coche y el crecimiento económico basado en el statu quo. Las políticas climáticas destinadas a promover el decrecimiento, regular la movilidad insostenible o el consumo de productos de origen animal chocan con estos ideales patriarcales, vulnerables ante el ataque a sus elementos identitarios.

Para enfrentar el problema climático - que es en esencia una derivada más del problema de sobrepoblación, sobreexplotación y envenenamiento del medio ambiente por la humanidad-, es necesario transitar hacia otras formas de ver el mundo más éticas, igualitarias y sostenibles, esto es, promoviendo aquellas visiones de cambio sistémico que realmente respondan a la situación de emergencia en que nos encontramos (Fernández-Reyes, 2013). Afirma Sara Ahmed (2019) que "señalar que el superdesarrollo nos está conduciendo a un vaciamiento del 
mundo es ser aguafiestas, interponerse en el camino de un disfrute futuro" (p. 357). Así, los movimientos transformadores basados en el decrecimiento, el antiespecismo o el feminismo de los cuidados, entre otros aspectos, no se enfrentan solo al problema climático, sino a toda una cultura y un sistema ideológico que sustenta la insostenible forma de vida occidental. Desde diferentes corrientes de pensamiento surgen propuestas destinadas a promover el cambio cultural que la crisis climática requiere, que consisten en adoptar una mirada antiantropocéntrica (Almiron y Tafalla, 2019; Best, 2014), ecofeminista (Gaard, 2015; Herrero, 2013), interseccional (Hathaway, 2020; Kaijser y Kronsell, 2014) y decrecentista (Keyßer y Lenzen, 2021; Taibo, 2011) sobre la crisis climática.

Reducir la discusión climática a "negacionistas vs no negacionistas" no es pues solo reduccionista y polarizador, es también una forma de invisibilizar este sustrato ideológico que subyace bajo la inacción climática de tantos no negacionistas del cambio climático. Una polarización que oculta en definitiva el papel que tiene el sistema capitalista antropocéntrico, patriarcal y basado en una idea de progreso centrada en el crecimiento industrial-tecnológico ilimitado. Es precisamente invisibilizar a los valores que nos han conducido a la situación actual y que no son solo patrimonio de una "derecha conservadora negacionista".

\section{COMUNICAR LA COMPLEJIDAD}

Comunicar la complejidad es una tarea difícil (requiere manejar muchas variables) e ingrata (no facilita los titulares $u$ acciones comunicativas simples, que son los que tienen más impacto y por tanto los más deseados). Para George Lakoff $(2010,2014)$ la dificultad de comunicar la complejidad radica en que los humanos pensamos en encuadres (frames), esto es, explicaciones particulares sobre un asunto basadas en un enfoque determinado sobre esa realidad. Pero las cuestiones complejas se resisten a ser tratadas con enfoques únicos, especialmente cuando, como es habitual, tienen causas sistémicas.

Comunicar la complejidad de la inacción climática no escapa a esta lógica, que viene además complicada por las estrategias de relaciones públicas del contramovimiento climático (Almiron y Xifra, 2020) y otros condicionantes estructurales que actúan sobre la labor periodística (Mancinas-Chávez, 2013; Moreno-Cabezudo, 2018). Sin embargo, al tratar de escapar de la simplificación, hay que tener cuidado de no complejizar más la cuestión. Algunas de las soluciones que Lakoff (2014) sugiere por ejemplo para abordar la complejidad del cambio climático -acudir a redes de causas interconectadas, ciclos de retroalimentación o cálculos probabilísticos- pueden llegar a complicar más que aclarar, lo cual paraliza la acción y alimenta el argumento del abandono (si es demasiado complejo, no hay nada que hacer, se tiende a pensar).

No podemos ofrecer aquí recomendaciones específicas para hacer bien esta tarea, para la que por otro lado no existen fórmulas mágicas (ver por ejemplo Boykoff, 2019), pero sí establecer algunas recomendaciones generales con respecto a la cuestión de la terminología para comunicar las causas de la inacción climática. Si nuestro objetivo es implementar una comunicación ética, igualitaria y sostenible al respecto, sugerimos tres reflexiones:

En primer lugar, es importante recordar que el episodio de cambio climático antropogénico actual no es más que la consecuencia de nuestro impacto medioambiental. Un impacto 
ambiental que causa enorme sufrimiento a muchos humanos y no humanos, siendo por lo general los más afectados entre los humanos las capas sociales que menos contaminan, las más empobrecidas, mientras que el total de no humanos es inocente de tal contaminación y la sufre por igual. Este recordatorio es relevante porque, desde una mirada ética y pragmática, deberíamos reconocer que no hace falta cambio climático para aceptar que contaminar es incorrecto, con o sin calentamiento global. En la medida en que ponemos esta postura ética en el foco comunicativo, se hace inmediatamente evidente la necesidad de promover la acción efectiva y abandonar la acción dilatoria. Como afirma Fernández-Reyes (2014), debemos dejar de centrar la comunicación en lo "que se dice" y centrarla en lo "que se hace" y "lo que ocurre". Precisamente lo contrario que pretenden las estrategias de las industrias más contaminantes, exquisitamente pro-medioambiente en público e intensamente obstructivas en lo político en la práctica. Los consensos sobre cuánto se contamina, cómo medir las emisiones, quién contamina más, cómo medir los impactos de la contaminación, etc. -que forman parte de la estrategia de dilación central de las principales industrias obstruccionistas-son una acción de relaciones públicas enmascarada como acción climática. La comunicación de la inacción climática debería tener muy en cuenta no cubrir como acción discursiva lo que solo es acción dilatoria.

En segundo lugar, el marco "negacionistas vs. no negacionistas" es un marco que debe ser abandonado como concepto global para explicar la inacción política. El negacionismo del cambio climático antropogénico existe, de forma substancial, en los Estados Unidos, pero no se trata esta de una posición que represente al conjunto de los actores causantes de la inacción climática ni es mayoritaria en la argumentación utilizada. Centrar la inacción climática en el negacionismo es reduccionista y maniqueo, contribuye a reforzar una falsa realidad que contrapone actores buenos contra actores malos. La inacción climática, en Europa, está principalmente impulsada por actores no negacionistas de la ciencia climática.

Por último, hemos propuesto en este artículo los principales conceptos que informan los diferentes matices de las causas de la inacción climática. Para ello, hemos hecho especial hincapié en el obstruccionismo, que refleja no "lo que dicen" sino "lo que hacen" y "lo que ocurre" de la inmensa mayoría de actores que, al margen de sus ideas, efectivamente se dedican a influir en la agenda pública, el proceso político y la opinión pública para que las acciones necesarias no se lleven a cabo y la ciudadanía apoye el statu quo, o cuanto menos, lo acepte y siga votando a esos mismos partidos políticos que se dejan influir. Términos como obstruccionismo, retardismo, oposición o contrarianismo no solo reflejan mejor la complejidad real del problema climático, sino que dejan de desviar la atención hacia un enemigo externo.

\section{NOTAS}

Este trabajo ha sido financiado por la Agencia Estatal de Investigación (AEI) y el Fondo Europeo de Desarrollo Regional (FEDER) (CSO2016-78421-R), y por el Ministerio de Ciencia, Innovación y Universidades (FPU18/04207).

\section{Referencias}

Adams, C. (2010). The sexual politics of meat. A feminist-vegetarian critical theory. The Continuum International Publishing Group.

Ahmed, S. (2019). La promesa de la felicidad. Caja Negra. 
Almiron, N. (2020). Meat taboo: Climate change and the EU meat lobby. En J. Hannan (Ed.), Meatsplaining: The Animal Agriculture Industry and the Rhetoric of Denial (pp. 163-186). Sidney University Press. https://doi.org/10.2307/j.ctv15tt7dr.11

Almiron, N., Boykoff, M., Narberhaus, M., y Heras, F. (2020). Dominant counter-frames in influential climate contrarian European think tanks. Climatic Change, 162(4), 2003-2020. https://doi.org/10.1007/s10584-020-02820-4

Almiron, N., y Moreno, J. A. (2021). Think tanks neoliberales y falsos debates: La propuesta del impuesto a la carne para combatir la crisis climática. En D. Rodrigo-Cano, R. Mancinas-Chávez, y R. Fernández-Reyes (Eds.), La comunicación del cambio climático, una herramienta ante el gran desafio (pp. 222-248). Dykinson. https://bit.ly/3pZrurF

Almiron, N., Rodrigo-Alsina, M., y Moreno, J. A. (2021). Manufacturing ignorance: Think tanks, climate change and the animal-based diet. Environmental Politics, 1-22. https://doi.org/10.1080/09644016.2021.1933842

Almiron, N., y Tafalla, M. (2019). Rethinking the Ethical Challenge in the Climate Deadlock: Anthropocentrism, Ideological Denial and Animal Liberation. Journal of Agricultural and Environmental Ethics, 32(2), 255-267. https://doi.org/10.1007/s10806-019-09772-5

Almiron, N., y Xifra, J. (2020). Climate Change Denial and Public Relations. Strategic Communication and Interest Groups in Climate Inaction. En Climate Change Denial and Public Relations. Routledge. https://doi.org/10.4324/9781351121798

Best, S. (2014). Moral Progress and the Struggle for Human Evolution. En The Politics of Total Liberation. Revolution for the 21st Century (pp. 87-98). Palgrave Macmillan.

Boykoff, M. (2016). Consensus and contrarianism on climate change: How the USA case informs dynamics elsewhere. Mètode Revista de difusió de la investigació, 6, 89-95. https://doi.org/10.7203/metode.85.4182

Boykoff, M. (2019). Creative (Climate) Communications: Productive Pathways for Science, Policy and Society (1. ${ }^{\text {a }}$ ed.). Cambridge University Press. https://doi.org/10.1017/9781108164047

Brulle, R. J. (2014). Institutionalizing delay: Foundation funding and the creation of U.S. climate change counter-movement organizations. Climatic Change, 122(4), 681-694. https://doi.org/10.1007/s10584-013-1018-7

Coan, T., Boussalis, C., Cook, J., y Nanko, M. (2021). Computer-assisted detection and classification of misinformation climate change [Preprint]. SocArXiv. https://doi.org/10.31235/osf.io/crxfm

Cohen, S. (2001). States of denial: Knowing about atrocities and suffering. Polity Press.

CSSN. (2021). The Structure of Obstruction: Understanding Opposition to Climate Change Action in the United States (CSSN Primer). Climate Social Sciences Network (CSSN). https://bit.ly/3GI10I3

de Freitas Netto, S. V., Sobral, M. F. F., Ribeiro, A. R. B., y Soares, G. R. da L. (2020). Concepts and forms of greenwashing: A systematic review. Environmental Sciences Europe, 32(1), 19. https://doi.org/10.1186/s12302-020-0300-3

Duerringer, C. (2015). Be a Man-Buy a car! Articulating masculinity with consumerism in «Man's last stand». Southern Communication Journal, 80(2), 137-152. https://doi.org/10.1080/1041794X.2015.1017654

Dunlap, R. E. (2013). Climate change skepticism and denial. American Behavioral Scientist, 57(6), 691698. https://doi.org/10.1177/0002764213477097 
Dunlap, R. E., y McCright, A. M. (2011). Organized climate change denial. En J. S. Dryzek, R. B. Norgaard y D. Schlosberg (Eds.), The Oxford handbook of climate change (pp. 144-160). Oxford University Press.

Dunlap, R. E., y McCright, A. M. (2015). Challenging climate change: The denial countermovement. En R. E. Dunlap y R. Brulle (Eds.), Climate change and society: Sociological perspectives (pp. 300-332). Oxford University Press.

European Parliament. (2017). Special Eurobarometer 459: Climate change. Directorate-General for Communication. https://bit.ly/322VN8h

Farand, C., Hope, M., y Collett-White, R. (2019, 7 de junio). Mapped: A who's who of Brexit and climate science denial. DeSmog. https://bit.ly/3m0yCmk

Fernández-Reyes, R. (2013). Reflexiones sobre un periodismo en transición. En R. Mancinas-Chávez y R. Fernández-Reyes (Eds.) Medios de comunicación y cambio climático. Actas de las Jornadas Internacionales, (pp. 249-272). Fenix Editora. https://bit.ly/30oOjMv

Fernández-Reyes, R. (2014). Infoxicación en la comunicación del cambio climático. Mitigación y adaptación. Ámbitos. Revista Internacional de Comunicación, 26, 66-76. https://doi.org/10.12795/ambitos.2014.i26.07

Gaard, G. (2015). Ecofeminism and climate change. Women's Studies International Forum, 49, 20-33. https://doi.org/10.1016/j.wsif.2015.02.004

Garcés, M. (2017). Nova il·lustració radical. Anagrama.

Hathaway, J. R. (2020). Climate change, the intersectional imperative, and the opportunity of the Green New Deal. Environmental Communication, 13-22. https://doi.org/10.1080/17524032.2019.1629977

Herrero, Y. (2013). Miradas ecofeministas para transitar a un mundo justo y sostenible. Revista de economía crítica, 16, 278-307.

Hribal, J. (2014). Los animales son parte de la clase trabajadora y otros ensayos. Ochodoscuatro. https://bit.ly/3EYTDp8

Hultman, M., y Pulé, P. M. (2018). Ecological Masculinities. Routledge. https://doi.org/10.4324/9781315195223

Huth, K., y Peters, J. (2020, 4 de febrero). Die Heartland lobby. Correctiv. https://bit.ly/3ypKrHK

Ivanova, D., Barrett, J., Wiedenhofer, D., Macura, B., Callaghan, M. W., y Creutzig, F. (2020). Quantifying the potential for climate change mitigation of consumption options. Environmental Research Letters, 15(9), 093001. https://doi.org/10.1088/1748-9326/ab8589

Jacques, P. J. (2012). A general theory of climate denial. Global Environmental Politics, 12(2), 9-17. https://doi.org/10.1162/GLEP_a_00105

Jacques, P. J., Dunlap, R. E., y Freeman, M. (2008). The organisation of denial: Conservative think tanks and environmental scepticism. Environmental Politics, 17(3), 349-385. https://doi.org/10.1080/09644010802055576

Jylhä, K. M., y Hellmer, K. (2020). Right-wing populism and climate change denial: The roles of exclusionary and anti-egalitarian preferences, conservative ideology, and antiestablishment attitudes. Analyses of Social Issues and Public Policy, 20(1), 315-335. https://doi.org/10.1111/asap.12203 
Kaijser, A., y Kronsell, A. (2014). Climate change through the lens of intersectionality. Environmental Politics, 23(3), 417-433. https://doi.org/10.1080/09644016.2013.835203

Keyßer, L. T., y Lenzen, M. (2021). $1.5^{\circ} \mathrm{C}$ degrowth scenarios suggest the need for new mitigation pathways. Nature Communications, 12(1), 2676. https://doi.org/10.1038/s41467-021-22884-9

Kramcksak Muñoz, X. C. (2021). ONGs ecologistas en España. Discurso, negacionismo ideológico y crisis climática. Revista Latinoamericana de Estudios Críticos Animales, 8(1), 151-183.

Lahsen, M. (2013). Anatomy of dissent: A cultural analysis of climate skepticism. American Behavioral Scientist, 57(6), 732-753. https://doi.org/10.1177/0002764212469799

Lakoff, G. (2010). Why it matters how we frame the environment. Environmental Communication, 4(1), 70-81. https://doi.org/10.1080/17524030903529749

Lakoff, G. (2014). Don't think of an elephant! (2. 'ed.). Chelsea Green.

Lamb, W. F., Mattioli, G., Levi, S., Roberts, J. T., Capstick, S., Creutzig, F., Minx, J. C., Müller-Hansen, F., Culhane, T., y Steinberger, J. K. (2020). Discourses of climate delay. Global Sustainability, 3, e17. https://doi.org/10.1017/sus.2020.13

Love, H. J., y Sulikowski, D. (2018). Of meat and men: Sex differences in implicit and explicit attitudes toward meat. Frontiers in Psychology, 9. https://doi.org/10.3389/fpsyg.2018.00559

Mancinas-Chávez, R. (2013). El silencio mediático. Reflexión en torno a las razones de los medios de comunicación para no hablar del cambio climático. En R. Mancinas Chávez y R. FernándezReyes (Eds.), Medios de comunicación y cambio climático (pp. 233-248). Fénix Editora. https://bit.ly/3oRjb1M

McCright, A. M. (2007). Dealing with climate change contrarians. En S. C. Moser y L. Dilling (Eds.), Creating a Climate for Change: Communicating Climate Change and Facilitating Social Change (pp. 200-212). Cambridge University Press.

McCright, A. M., y Dunlap, R. E. (2010). Anti-reflexivity. The American Conservative Movement's Success in Undermining Climate Science and Policy. Theory, Culture \& Society, 27(2-3), 100-133. https://doi.org/10.1177/0263276409356001

McCright, A. M., y Dunlap, R. E. (2011). Cool dudes: The denial of climate change among conservative white males in the United States. Global Environmental Change, 21(4), 1163-1172. https://doi.org/10.1016/j.gloenvcha.2011.06.003

McKie, R. E. (2017). Rebranding the climate change counter movement: A critical examination of counter movement messaging through a criminological and political economic lens [Tesis doctoral]. https://dora.dmu.ac.uk/handle/2086/15355

McKie, R. E. (2021). Climate change obstruction, criminal negligence and ecological collapse. The Peace Chronicle. The Magazine of the Peace and Justice Studies Association. https://bit.ly/3dVp0ok

Mies, M. (2018). Patriarcado y acumulación a escala mundial. Traficantes de Sueños.

Moreno, J. A., y Ruiz-Alba, N. (2021). ¿Periodismo o greenwashing? Patrocinadores de la COP25 ChileMadrid en la prensa española. Revista Mediterránea de Comunicación, 12(2), 285-300. https://www.doi.org/10.14198/MEDCOM.19089

Moreno-Cabezudo, J. A. (2018). Estructura informativa y Cambio Climático: El caso de «El País». En R. Fernández-Reyes y D. Rodrigo-Cano (Eds.), La comunicación de la mitigación y la adaptación al Cambio Climático (pp. 77-98). Egregius. https://bit.ly/3pUsc9u

Norgaard, K. M. (2011). Living in denial: Climate change, emotions, and everyday life. MIT Press. 
O'Neill, S. J., y Boykoff, M. (2010). Climate denier, skeptic, or contrarian? Proceedings of the National Academy of Sciences, 107(39), E151-E151. https://doi.org/10.1073/pnas.1010507107

Oreskes, N., y Conway, E. M. (2018). Mercaderes de la duda. Cómo un puñado de científicos ocultaron la verdad sobre el calentamiento global. Capitán Swing.

Piltz, R. (2008). The Denial Machine. Index on Censorship, 37(4), 72-81. https://doi.org/10.1080/03064220802561366

Rekker, R. (2021). The nature and origins of political polarization over science. Public Understanding of Science, 30(4), 352-368. https://doi.org/10.1177/0963662521989193

Rothgerber, H. (2013). Real men don't eat (vegetable) quiche: Masculinity and the justification of meat consumption. Psychology of Men \& Masculinity, 14(4), 363-375. https://doi.org/10.1037/a0030379

Supran, G., y Oreskes, N. (2017). Assessing ExxonMobil's climate change communications (1977-2014). Environmental Research Letters, 12(8), 084019. https://doi.org/10.1088/1748-9326/aa815f

Supran, G., y Oreskes, N. (2021). Rhetoric and frame analysis of ExxonMobil's climate change communications. One Earth, S2590332221002335. https://doi.org/10.1016/j.oneear.2021.04.014

Taibo, C. (2011). En defensa del decrecimiento. Sobre capitalismo, crisis y barbarie. Catarata.

Walker, L., Butland, D., y Connell, R. (2000). Boys on the road: Masculinities, car culture, and road safety education. The Journal of Men's Studies, 8(2), 153-169. https://doi.org/10.3149/jms.0802.153

About the authors

Núria Almiron es profesora titular del Departamento de Comunicación de la Universitat Pompeu Fabra de Barcelona, donde forma parte del Critical Communication Research Group (CRITICC). Es directora del proyecto de investigación THINKClima (CSO2016-78421-R), codirectora del Center for Animal Ethics-UPF y directora del Máster en Estudios Internacionales sobre Medios, Poder y Diversidad de la misma universidad. Su investigación se centra en la comunicación y la economía política en ámbitos como la ética animal y el negacionismo del cambio climático.

Jose A. Moreno es investigador predoctoral del programa de doctorado en Comunicación de la Universitat Pompeu Fabra de Barcelona dentro del Programa de Formación del Profesorado Universitario (FPU) del Ministerio de Ciencia, Innovación y Universidades. Miembro del Critical Communication Research Group (CRITICC) y del proyecto de investigación THINKClima (CSO2016-78421-R) en el Departamento de Comunicación de la Universitat Pompeu Fabra. Su línea de investigación se centra en la comunicación sobre el cambio climático y los grupos de interés. 